\section{Statin therapy discontinuation increases risk of death in survivors of ischemic stroke}

Interruption of long-term statin therapy is detrimental in patients with acute myocardial infarction, but the clinical effect of therapy discontinuation in patients with ischemic stroke is not known.

Colivicchi et al. prospectively studied 631 consecutive survivors of acute ischemic stroke (mean age 70.2 \pm 7.6 years) who had no evidence of coronary heart disease. All patients were discharged from a single institution during a 4.5-year period and were prescribed either atorvastatin $(77.6 \%$ of individuals; mean dose $17.5 \pm 4.3 \mathrm{mg} /$ day) or simvastatin $(22.4 \%$ of individuals; mean dose $24.7 \pm 8.5 \mathrm{mg} /$ day). Patient adherence to therapy was assessed by telephone interview at 1, 6 and 12 months.

During the 12-month follow-up period, 246 (38.9\%) patients discontinued statin therapy (mean time to discontinuation $48.6 \pm 54.9$ days). Mild adverse effects were the cause of discontinuation in nearly a third of these patients; however, in the remainder of cases neither the patient nor the primary care physician could provide a specific medical reason for statin discontinuation. The primary end point of death from any cause occurred in $116(18.4 \%)$ patients (1-year probability of death $0.18,95 \% \mathrm{Cl} 0.15-$ 0.21 ); cardiovascular events were considered the cause of death in $93(80.1 \%)$ of these cases. Statin discontinuation was an independent predictor of 12-month all-cause mortality (hazard ratio $2.78,95 \% \mathrm{Cl} 1.96-3.72 ; P=0.003)$, as were age, stroke severity on admission, and discontinuation of antiplatelet therapy.

The authors conclude that patient care in the transition from a hospital to an outpatient setting should be improved in an effort to decrease the risk of death associated with discontinuation of statin therapy.

Original article Colivicchi F et al. (2007) Discontinuation of statin therapy and clinical outcome after ischemic stroke. Stroke 38: 2652-2657

\section{Encouraging increase in the use of medical therapy in patients hospitalized with AMI}

Large randomized trials have definitively proven the effectiveness of aspirin, $\beta$-blockers, angiotensin-converting-enzyme inhibitors and statins for secondary prevention in patients discharged from hospital after acute myocardial infarction (AMI). Consequently, guidelines for the treatment of patients after AMI recommend routine use of these therapies. To determine the current and changing trends in the prescription of these drugs, Goldberg et al. conducted a multinational observational study.

By use of data from the Global Registry of Acute Coronary Events (GRACE), the researchers identified 26,413 patients with $\mathrm{AMI}$ and without contraindications to the four medications examined who were hospitalized within the period January 2000 to December 2005. Over this period, marked increases were noted in the percentages of patients prescribed statins (from $45 \%$ to $85 \%$ ) and angiotensin-converting-enzyme inhibitors (from 63\% to $77 \%$ ). The proportion of patients prescribed $\beta$-blockers also increased (from $83 \%$ to $91 \%$ ), but little increase was noted in the use of aspirin, as prescription of this drug was already high ( 95\%) in 2000. The percentage of patients prescribed all four medications increased considerably over the study period, rising from $\sim 23 \%$ of patients in the first 6 months of 2000 , to $~ 58 \%$ of patients in the last 6 months of 2005. Almost identical trends in the use of single and combination therapy were seen in patients with ST-segment and non-ST-segment elevation AMI. Age $\geq 65$ years, female sex, history of heart failure or stroke and the development of atrial fibrillation during hospitalization were all associated with the underuse of combination therapy.

\section{Original article Goldberg RJ et al. (2007) Increasing use of single and combination medical therapy in patients hospitalized for acute myocardial infarction in the 21st century: a multinational perspective. Arch Intern Med 167: 1766-1773 \\ Value of self-reported medication adherence in patients with coronary heart disease}

Nonadherence to prescribed medications by patients with coronary heart disease (CHD) is associated with an increased incidence of adverse cardiovascular events, but it is not known whether self-reported nonadherence can identify patients at greatest risk of such events.

Gehi et al. enrolled 1,024 outpatients with documented stable CHD and assessed the 